\title{
Distinct striatal subregions and corticostriatal connectivity for effort, action and reward
}

$17{ }^{1}$ Translational Research in Affective Disorders Laboratory, Department of Psychology, Emory University, 18 Atlanta, GA

$19{ }^{2}$ Department of Psychiatry and Behavioral Science, Emory University, Atlanta, GA

25 Correspondence to:

26 Michael T. Treadway

27 Department of Psychology

28 Emory University

29 Atlanta, GA 30306

30 p: 404-727-3166

31 e:mtreadway@emory.edu 


\begin{abstract}
The ventral striatum is believed to encode the subjective value of cost/benefit options; however, this effect has strikingly been absent during choices that involve physical effort. Prior work in freely-moving animals has revealed opposing striatal signals, with greater response to increasing effort demands and reduced responses to rewards requiring effort. Yet, the relationship between these conflicting signals remains unknown. Using fMRI with a naturalistic, effort-based navigation paradigm, we identified functionally-segregated regions within ventral striatum that separately encoded action, effort, and discounting of rewards by effort. Strikingly, these sub-regions mirrored results from a large-sample connectivity-based parcellation of the

42 striatum. Moreover, individual differences in striatal effort activation and effort discounting 43 signals predicted striatal responses to effort-related choices during an independent fMRI task. 44 Taken together, our results suggest that a dorsomedial region primarily associated with action may instead represent the effort cost of actions, and raises fundamental questions regarding the interpretation of striatal "reward" signals in the context of effort demands.
\end{abstract}

34 45

47

Keywords: effort discounting, value-based decision-making, fMRI, naturalistic stimuli

49

50

51

52 


\section{Introduction}

Weighing the costs and benefits of actions is critical for everyday decisions. The ventral striatum (VS) is widely recognized as a central hub for processing cost/benefit information ${ }^{1-3}$.

This role for VS has been supported by robust findings from animal models implicating striatal dopamine (DA) signals in valuation and motivating actions for rewards ${ }^{4-8}$. Building upon the rich preclinical literature, human neuroimaging studies have yielded relatively homogenous results regarding the involvement of the VS in encoding subject-specific, discounted value signals ${ }^{1}$. For example, fMRI studies have demonstrated that VS responses not only are consistently greater for immediate compared to delayed rewards ${ }^{9-11}$, but also explicitly track the delay-discounted subjective value rather than the objective magnitude of rewards ${ }^{12-14}$. Similarly, VS activity is strongly associated with expected reward such that less probable rewards elicit decreased responses ${ }^{15,16}$, and tracks with the probability-discounted subjective value ${ }^{13}$. Moreover, it has been proposed that VS encodes subjective value signals that are domain-general, such that different types of cost/benefits are incorporated and represented on a common scale ${ }^{17}$. Consistent with this, VS has been shown to track subjective value regardless of whether the reward was probabilistic or delayed ${ }^{13}$. Thus, the representation of subjective value signals in the VS detected during cost/benefit decision-making has been largely consistent with the vast preclinical literature highlighting the role of striatal signaling in valuation.

A surprising exception has been the neural representation of costs related to effort (i.e., the amount of work required to obtain rewards). Researchers have used various effort-based decision-making paradigms to decode effort-related value signals ${ }^{14,18-31}$ with widely varying study designs (e.g., timing at which effort/reward information is presented, type and magnitude of effort demands, inclusion of effort performance during the task, inclusion of a learning 
77 component). Critically, a majority of these studies did not find a significant association between

78 subjective value and VS activity ${ }^{14,19-21,23-25,27-30}$, not only in humans but also in rodents ${ }^{32}$. This

79 observation is strikingly at odds with prevailing theories about the functional significance of VS,

80 and discordant with consistent patterns of value-related VS activity found in other forms of

81 cost/benefit decision-making ${ }^{12-14}$ as well as evidence demonstrating that disrupting the VS

82 reliably impairs the willingness to work for rewards ${ }^{7,33,34}$.

Recent work examining striatal function in freely-moving animals suggests one potential

84 resolution to this discrepancy ${ }^{35-38}$. Such studies have found that DA inputs to the striatum signal

85 the initiation of vigorous action ("effort activation") ${ }^{38}$, while also representing the value of cost/benefit choices that are discounted by effort-related costs ("effort discounting") ${ }^{6,36}$. These

87 observations raise the possibility that detection of effort-discounting signals may perhaps be hindered by the simultaneous presence of an effort-activation signal in most neuroimaging paradigms, and that critical aspects of striatal function may only be revealed by carefully parsing these opposing influences.

Importantly, these action- and value-related signals associated with effort demands may

92 arise from distinct neural populations within the striatum ${ }^{39,40}$. For example, prior work examining the neural substrates of goal-directed behavior has suggested that morphological subregions of

94 the VS, including the nucleus accumbens (NAcc) core and shell ${ }^{41}$, have different functions that 95 together guide actions for rewards ${ }^{2,42}$. Such studies have provided evidence suggesting that 96 neighboring regions within the VS have separable roles in processing reward- and effort-related 97 information ${ }^{43-46}$. In a separate line of work, striatal subregions have also been identified by 98 parcellating the striatum based on intrinsic functional connectivity MRI (fcMRI) ${ }^{47}$, although the 99 functional significance of these subregions remain unclear. Importantly, these reported 
100

101

102

103

104

105

106

107

108

109

110

111

112

113

114

115

116

117

118

119

120

121

122

morphological and connectivity-based striatal organizations raise the hypothesis that effort activation and discounting signals may originate from different subregions within VS; however, this has previously not been tested.

Additionally, the importance of including a naturalistic action component within experimental paradigms is highlighted by evidence suggesting that (i) midbrain DA activity controls the initiation of future action ${ }^{35}$ and (ii) reward-related striatal DA inputs are attenuated in the absence of action initiation ${ }^{38}$. To date, however, most studies investigating the neural mechanisms of effort-based choice in humans have commonly focused on evaluating neural responses during the presentation or outcome of effort- or reward-related cues or choices_-but not during periods of effortful action — as the primary method with which to identify the neural substrates of subjective value $\mathrm{e}^{14,18-31}$. More importantly, these prior studies have not been designed to clearly distinguish effortful or vigorous action from mere movement. This raises fundamental questions regarding the interpretation of striatal "reward" signals given the potentially-critical role of dynamic action. Notably, it has been demonstrated that naturalistic action within virtual-reality paradigms can invoke midbrain DA neuron activity in the mouse brain $^{48}$, offering a means by which this preclinical work can be translated to human neuroimaging.

Here, we combined functional magnetic resonance imaging (fMRI) with virtual navigation to evaluate effort-, action-, and reward-related activity in the human striatum. Specifically, participants navigated through 3D mazes with the objective of obtaining rewards (Fig. 1A), and completion of the mazes varied across four conditions: Two in which individuals actively controlled navigation using (i) high-effort and (ii) low-effort button pressing, and two in which individuals passively completed navigation, one (iii) with motion and the other (iv) 
123

124

125

126

127

128

129

130

131

132

133

without motion. Neural activity during the maze-navigation task was additionally examined in relation to neural responses during a second effort based-benefit decision-making task, which was independently assessed using a paradigm previously published by our group ${ }^{30}$. During this second task, participants made choices about their willingness to perform manual button presses for monetary rewards. As such, we were able to examine neural responses to periods of effort activation and effort discounting, and then see how they contributed to neural responses when making decisions about the value of work.

\section{Results}

Activity in ventral striatal subregions are modulated by anticipation and initiation of vigorous action

To examine whether the VS encoded effort activation in the maze-navigation task, we compared hemodynamic response signals between a High-Effort condition that required rapid and repeated button-pressing to move through the maze, and a Low-Effort condition that allowed individuals to simply hold down buttons to move. This signal was compared at two separate timepoints: (i) when participants learned about the required effort (i.e., anticipation), and (ii) when participants began execution of action (i.e., initiation). Indeed, both the anticipation and initiation of High- relative to Low-Effort increased activity in a dorsal subregion of the VS extending up into dorsomedial striatum and caudate body (MNI coordinates [x,y,z], anticipation: left sub-peak: -12, 0, 15; right sub-peak: 12, 12, 6; initiation: left sub-peak: $-9,0,6$; right subpeak: 9, 6, -3; Fig. 1B). Notably, this effort-related dorsomedial subregion of VS (“dmVS”) was not modulated by the anticipation or initiation of action alone (i.e., during low-effort navigation), as evaluated by comparing the Low-Effort condition to a Passive-Motion condition that involved automatic progression through the maze without any required finger movement. Instead, action 
147 initiation strongly recruited a distinct striatal subregion in bilateral putamen for both the High-

148 and Low-Effort conditions (left sub-peak: -24, 0, -6; right sub-peak: 24, 3, -3). In a follow-up

149 region-of-interest (ROI) analysis using independently-defined ROIs (see Methods), we found

150 evidence for a double-dissociation such that responses within the dmVS (Fig. 1C) and putamen

151 (Fig. 1D) were selective to effort and action, respectively (Region*Effect interaction:

$\left.152 \quad F_{(1,28)}=43.34, p<0.001, \eta^{2}=0.61\right)$.

153

Effort discounting is represented in ventral striatal activity during reward receipt

155

We then examined whether VS also encoded effort discounting. Upon completion of

mazes, participants were presented with a reward amount $(\$ 0.00-\$ 5.00)$ that varied

157 independently of the navigation condition. An anterior/ventral subregion of VS (“aVS")

158 responded to reward receipt (left sub-peak: $-3,15,-3$, right sub-peak: 6, 15, -3), and was

159 positively correlated with trial-by-trial reward magnitude (left sub-peak: -6, 18, -6; right sub-

160 peak: $6,15,-3)$ across all navigation conditions, including a No-Motion condition that involved

161 simply waiting for the approximate duration of the maze. Importantly, while aVS exhibited a

162 reward response within the High-Effort condition, aVS response to reward was significantly

163 lower after effortful navigation compared to navigation that required no effort $\left(t_{(28)}=-1.88\right.$,

$164 p=0.03$; Fig. 1E), suggesting evidence of effort discounting. To further isolate effort discounting

165 in the VS, we examined whether there were areas of VS that responded to reward only during the

166 High-Effort condition. This analysis isolated a region of VS that was at the intersection of aVS

167 and dmVS, suggesting a node of interaction between effort activation and effort discounting (Fig.

$1681 \mathrm{~F}$; peak: $6,0,-9, k=72$ ). 
A
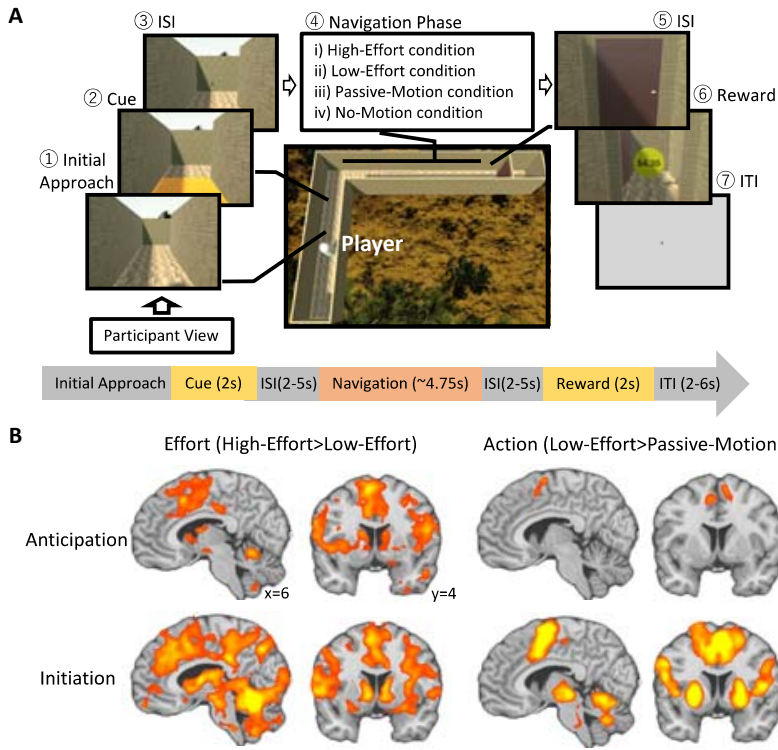
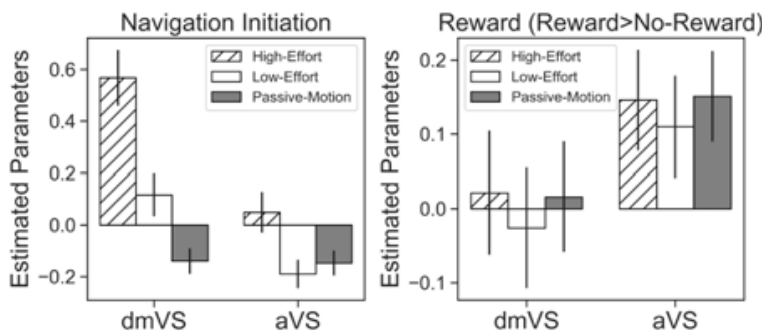

D

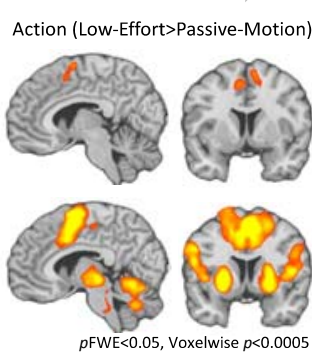

E
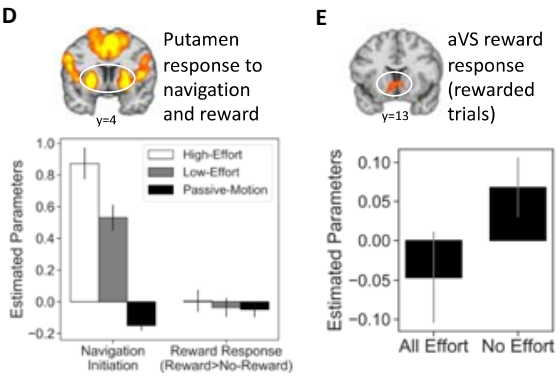

F

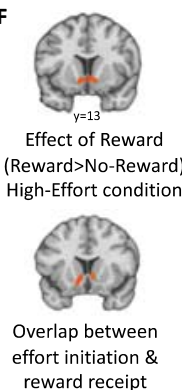

Fig. 1. Maze-navigation task schematic and results. (A) Schematic of a task trial. (B) Whole-brain results examining the effect of effort and action during anticipation and initiation of navigation. Maps are $p<0.05$ familywise-error ( $p$ FWE) cluster-corrected with voxel-threshold $p<0.0005$. (C) Independently-defined ROI analyses showing functional dissociation between the effort- and reward-related VS subregions. (D) Putamen response to navigation and reward. (E) Comparison of reward effects in aVS between effortful (High-/Low-Effort) and noneffortful conditions (Passive-/No-Motion). (F) VS response to reward after effortful navigation and overlap between effort initiation and reward effects.

Functional segregation of effort-, reward-, and action-related striatal subregions recapitulate connectivity-based parcellation of the striatum

Mapping together the above results (Fig. 2A), we observed functional segregation of bilateral striatal subregions associated with effort activation (dmVS), reward (aVS), and action (putamen). Critically, the neighboring regions associated with effort and reward showed clear evidence of double dissociation (Region*Condition interaction: $F_{(1,28)}=13.77, p<0.001, \eta^{2}=0.33$ ), with dmVS responding strongly to the anticipation and initiation of effort, but not receipt of reward, while aVS responded strongly to the magnitude of reward and appeared to discount reward values by effort, but showed significantly less engagement to anticipation or initiation of effortful action (Fig. 1C; also see Fig. S1 for results using unsmoothed data). Strikingly, this functional segregation appeared to largely overlap with a previously-reported connectivity-based 
189

190

191

192

193

194

195

196

197

parcellation of the striatum (Fig. $2 \mathrm{~A})^{47}$. To validate the apparent functional distinction between

these two VS subregions, we compared patterns of functional connectivity during resting-state

using each of the functionally-defined and connectivity-based striatal subregions as seed regions.

Indeed, despite only partial overlap of the ROI definitions derived from our task and the prior

parcellation study ${ }^{47}$ (Sørensen-Dice: aVS: 0.19; putamen: 0.40; dmVS: 0.20), we observed

highly overlapping connectivity profiles (Fig. 2B), suggesting that these ventral striatal

subregions participate in distinct functional networks. These connectivity profiles were

additionally replicated in an independent resting-state dataset collected with high-resolution (7-

Tesla) fMRI (Fig. 2B $)^{49}$.

A
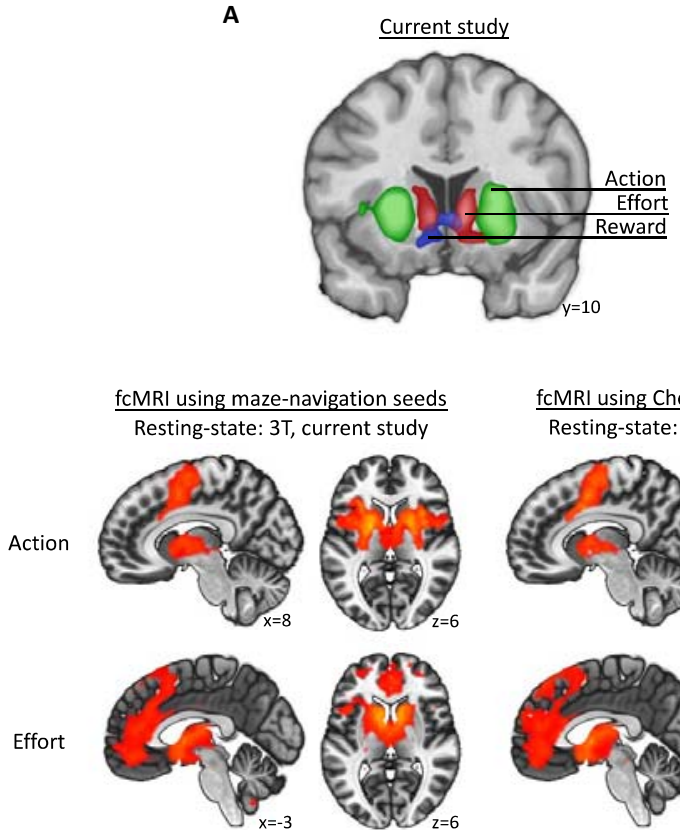

Reward

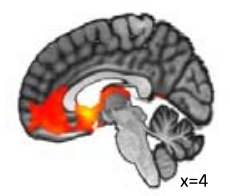

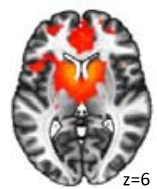

fcMRI using Choi et al 2012 seeds
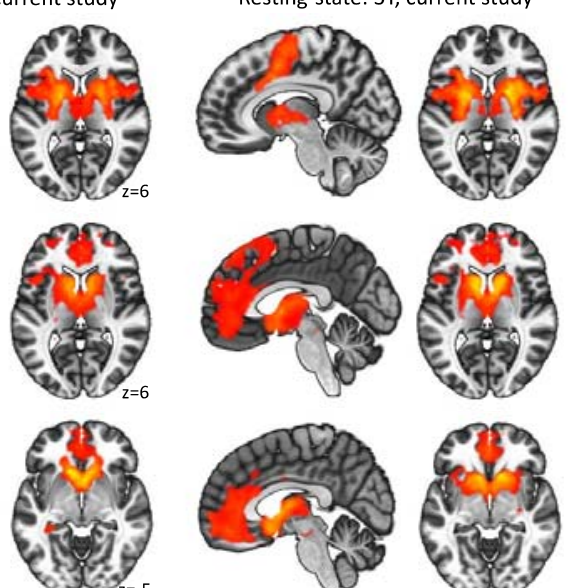

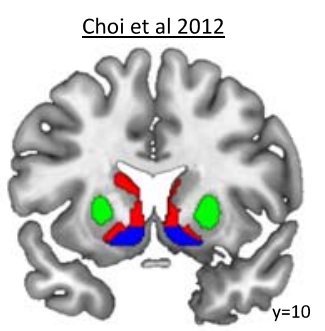

fcMRI using maze-navigation seeds

Resting-state: 7T, Gorgolewski et al 2015
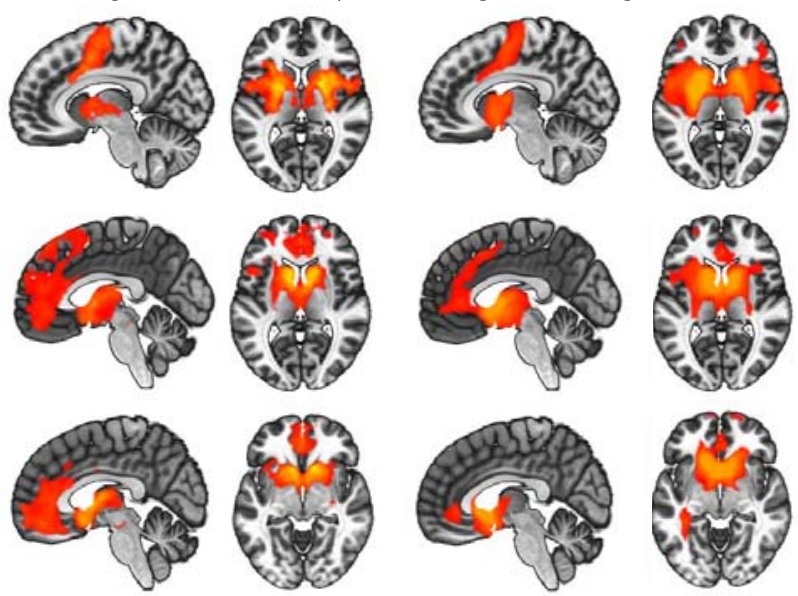

Fig. 2. Functional segregation of striatum and comparison of connectivity profiles with previously-identified striatal parcellation based on intrinsic connectivity. (A) Striatal subregions identified in the current study and corresponding subregions identified via connectivity-based parcellation ${ }^{47}$. (B) Comparison of seed-to-voxel functional connectivity maps using striatal regions in (A) as seed regions and resting-state data from the current study and prior work ${ }^{49}$. Maps are $p<0.05$ familywise-error corrected with voxelwise $p<0.0005$. 


\section{Lack of ventral striatal activity related to value during effort-based decision-making paradigm}

To test the hypothesis that effort activation and discounting signals may interfere with the detection of subjective signals during effort-based decision-making, we also measured neural activity during a second fMRI paradigm in which participants made a series of binary choices based on the presented amount of reward and effort required for each option (Fig. 3A). Importantly, the effort and reward amounts were presented sequentially in attempt to isolate an effort-activation signal during the anticipation of various effort demands. Replicating our previous findings using this same task ${ }^{30}$ as well as results from other fMRI effort-based paradigms, we did not find an association between VS activity and subjective value of the chosen option (Fig. 3B; Table S2). Even when using multivoxel pattern analysis, voxels in the VS were unable to classify whether presented information reflected reward or effort information (Fig. S2). In contrast, classifiers trained on activity across all brain voxels successfully decoded whether reward or effort information was presented for $78.9 \%$ of individuals (Fig. S3). As described above, the absence of such an effect is surprising, given the robust activation of VS by subjective values derived from cost/benefit decisions involving other categories of response costs (e.g., delay, risk, or loss). 


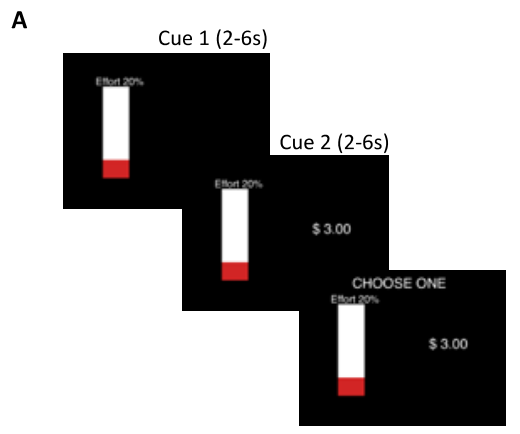

B

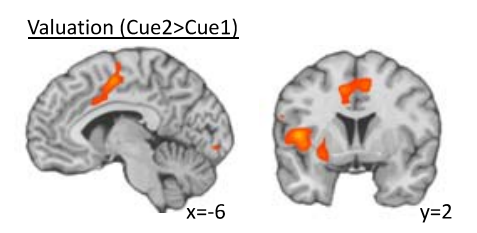

Subjective Value of Chosen Option during Cue 2

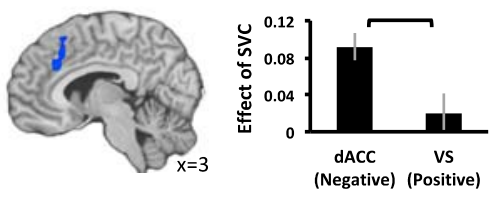

C

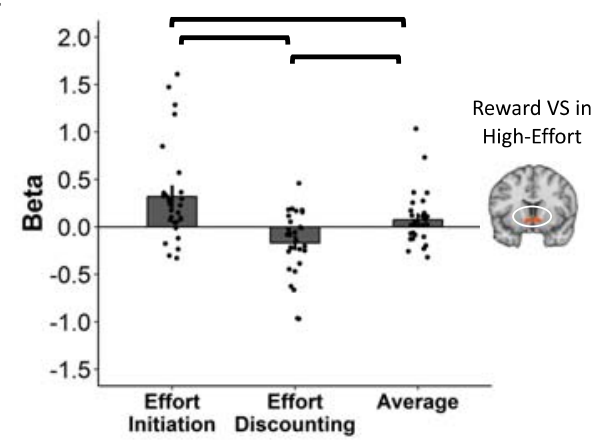

D

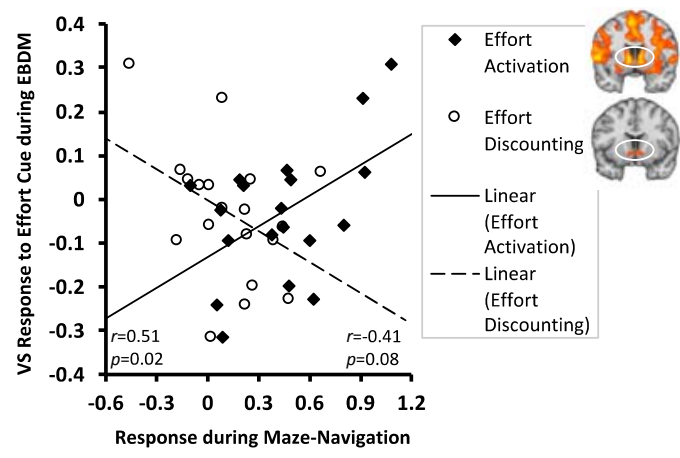

Fig. 3. Schematic and results of effort-based decision-making task and cross-paradigm analyses. (A) Schematic of a task trial. (B) Whole-brain results replicate our prior findings using this paradigm ${ }^{30}$, including activation of dorsal anterior cingulate cortex (dACC) and insula at Cue 2, association between dACC and negative subjective value, and absence of VS response across contrasts. Maps are $p<0.05$ familywise-error corrected with voxelwise $p<0.0005$. (C) aVS encodes both effort activation (High-Effort $>$ Low-Effort at navigation initiation) and effort discounting (High-Effort>Low-Effort at reward receipt) that average to a null-group effect. (D) Effort-related striatal signals during maze-navigation predict VS responses to effort information during effort-based decisionmaking (EBDM). Error bars in bar plots represent standard error of the mean.

Ventral striatal activity is modulated by opposing effects of effort initiation and effort discounting

One possible explanation for the absence of effort-related subjective-value encoding in VS is that when response costs involve physical effort, opposing effort-activation and effortdiscounting signals within VS may impede the ability to detect overall VS responses. To test this possibility in the maze-navigation task, we tracked responses within the VS region that responded to reward after individuals completed effortful navigation (Fig. 1F). This revealed two distinct patterns of effort-related activity in the VS depending on the timepoint, as evidenced by 
a significant Condition (High-Effort, Low-Effort)*Phase (navigation initiation, reward receipt) interaction $\left(F_{(1,28)}=16.72, p=0.0003, \eta^{2}=0.37\right)$ : VS responses were (i) significantly greater when individuals began executing effortful action compared to mere action $\left(t_{(28)}=3.42, p=0.002\right)$, reflecting an effort-activation signal, and (ii) significantly lower during reward receipt after individuals have expended effort compared to less effort $\left(t_{(28)}=-2.66, p=0.01\right)$, reflecting an effort-discounting signal. Importantly, averaging the opposing effects associated with effort activation and effort discounting within individuals led to a null group-level effect $(p=0.16$; Fig. $3 C)$.

However, such "averaging” is clearly artificial, as these task conditions did not occur simultaneously. A much stronger test would therefore be to show a mix of effort activation and effort discounting signals in response to the same effort-related stimulus. We therefore extracted the "effort activation" and "effort discounting" signals from the maze-navigation task, and used them as predictors of neural responses to effort cues in our second (independent) task. Interestingly, individual differences in effort-activation signal were positively associated with VS responses during presentation of effort information during decision-making $\left(r_{(17)}=0.51\right.$, $p=0.02$ ), whereas individual differences in effort-discounting signals exhibited the opposite pattern $\left(r_{(17)}=-0.41, p=0.08\right.$; Steiger's $Z=2.88, p=0.004$; Fig. 3D). These findings provide a potential explanation for the longstanding discrepancy regarding the involvement of VS in encoding subjective value during effort-based decision-making epochs, as such epochs might reasonably be expected to elicit both effort-discounting and effort-activation signals. 


\section{Discussion}

For almost two decades, neural responses in the ventral striatum have been commonly observed in response to rewards and discounted value of rewards. Thus, it has been surprising that most functional imaging studies in humans and animals have shown weak or inconsistent activation of VS during effort-based choice ${ }^{14,18-31}$. One methodological gap in prior studies is the

267 failure to take into consideration striatal signals during effortful action that have been detected under free-movement conditions in rodents ${ }^{35,38,50,51}$, which may impact the interpretation of reward-related striatal BOLD activity. Such studies have highlighted the presence of striatal

270 signals that are heavily linked to dynamic movement ${ }^{35,38}$, which can also be evoked by

271 movement within virtual-reality paradigms ${ }^{48}$. Thus, the present study used a virtual navigation

272 paradigm and revealed the presence of opposite signals in the VS related to (i) effort activation

273 during the anticipation and initiation of effortful action and (ii) effort discounting during reward

274 receipt after individuals expended effort. Importantly, the current study finds that effortful action

275 is needed to isolate these effects in the VS, as our second fMRI paradigm that relied solely on

276 effort- and reward- cues failed to differentiate them, even when using more sensitive multivariate

277 techniques. This observation is critical as prior studies of effort-based decision-making have

278 largely focused on encoding of values at the point of choice or in response to feedback ${ }^{14,19-21,23-}$

$272^{25,27-30}$. Critically, by using individual differences in estimates of effort-activation/effort-

280 discounting signals derived from the maze navigation task, we were able to observe their

281 simultaneous, conflicting influences when processing effort-related cues, providing a viable

282 explanation to the general failure to detect clear subjective-value signals in the VS during effort-

283 based decision-making in these prior work. Specifically, our results suggest that increased effort 
284

285

286

287

288

289

290

291

292

293

294

295

296

297

298

299

300

301

302

303

304

305

306

requirements may invoke a greater effort-activation response that may obscure detection of the value-related effort-discounting effect in the VS.

Additionally, the current results provide insight into the functional topography of signals associated with effortful movement and reward. Despite the rich body of research implicating midbrain DA in representing value-related information and invigorating movement ${ }^{4}$, little work has been done to simultaneously examine the striatal representation of these two functions in humans. Here, we identified an aVS region associated with reward, a neighboring dmVS region associated with effort-initiation, and bilateral putamen associated with initiation of simple, loweffort action. Interestingly, we observed an invigoration effect in $\mathrm{dmVS}$ during the anticipation and initiation of high- but not low-effort, suggesting that mere action (i.e., making a single choice response) may not be sufficient to shape VS signaling - further highlighting the importance of studying the neural substrates of effort-based decision-making that include effortful action components.

To our knowledge, the VS subregions identified here have not been previously characterized, though their existence is supported by prior large-scale connectivity results ${ }^{47}$. Indeed, we found overlap in functional-connectivity profiles between the striatal subregions identified here and the connectivity-based striatal parcellation ${ }^{47}$. Critically, this finding helps uncover potential functions of these network-based subregions of the striatum. Our results also show similarity to the pattern of results of a recent meta-analysis of subjective-value encoding ${ }^{1}$, where positive subjective value across studies was found to scale with activity in aVS, whereas negative subjective value (i.e., loss or punishment) was associated with activity in an area similar to dmVS. As activity in dmVS was only apparent as a function of effort but not action, whereas motor areas of putament appeared to respond to both increased effort and mere action, it raises 
307 the intriguing possibility that $\mathrm{dmVS}$ is more specifically involved with encoding negative value

308 or cost associated with effortful actions. Finally, it is worth noting that similar functional

309 heterogeneity within the ventral striatum has been observed in animal models [floresco].

310 Electrical recordings in non-human primates have found that distinct neuronal populations within

311 the striatum may be responsible for coding action and outcomes ${ }^{39,40}$. In rodents, while both the

312 NAcc core and shell are involved in Pavlovian learning of stimulus and outcome (e.g., cue and

313 reward), they play markedly different roles in terms of mediating Pavlovian influences on

314 instrumental behavior ${ }^{42,52}$.

315 Taken together, our results suggest that dmVS activity at the initiation of effortful action

316 is distinctly associated with neural representations of effortful action relative to reward-

317 responsive aVS. Importantly, our results suggest that simultaneous and conflicting influences of

318 invigoration and value in these neighboring regions may confound the absence of subjective-

319 value signals in prior work on effort-based decision-making. It is further notable that these

320 distinct sub-regions of ventral striatum were only observable when using a naturalistic paradigm,

321 echoing recent discoveries of striatal DA function found in freely-moving animals ${ }^{35-38}$. In sum,

322 these data will help advance our understanding of how the ventral striatum distinctly encodes

323 effort-related costs, and highlights the value of naturalistic and dynamic paradigms for achieving

324 a deeper understanding of real-world brain function ${ }^{53}$. 
328 This work was supported by funding from the NIMH R00 MH102355 and R01 MH108605 to

329 MTT and F32 MH115692 to JAC, and the National Science Foundation Graduate Research

330 Fellowship Program DGE- 1444932 to ARA. The authors would like to thank Johsua Buckholtz

331 and Daniel Dilks for helpful discussion and commentary. The authors additionally thank Brittany

332 DeVries, Makiah Nuutinen, Emma Hahn, Danielle Harrison, Annabel Lu, Maryam Rehman,

333 Jeffrey Yang, Kristi Kwok, Samuel Han, and Nimra Ahad for their assistance in data collection.

\section{AUTHOR CONTRIBUTIONS}

336

337

338

339

340

341

342

343

344

345

346

347

348

349

350

351

352

353

354

\section{DECLARATION OF INTERESTS}

The authors report no conflicts of interest, financial or otherwise. In the past three years, MTT has served as a paid consultant to Blackthorn Therapeutics and Avanir Pharmaceuticals. None of these entities supported the current work, and all views expressed herein are solely those of the authors.

SS wrote the first draft of the paper; SS and MTT designed the research; SS performed the research; SS, VL, JAC, ARA, and MTT analyzed the data; SS, VL, JAC, ARA, and MTT wrote the paper. 


\section{Methods}

All procedures were approved by the Emory Institutional Review Board. We recruited 30 healthy adults from the Atlanta community through fliers and online advertisements. Eligibility was determined using an online pre-screening survey. Participants were eligible if they were right-handed, English-speaking individuals between the ages of 18 and 35. Exclusion criteria included: (1) MRI contraindications (e.g., claustrophobia, metallic implants, CNS diseases, pregnancy in females); (2) Current use of psychoactive medications, investigational drugs, or those that affect blood flow (e.g., for hypertension); and (3) Current medical, neurological, or psychiatric illnesses.

Participants first provided informed consent and completed the MRI safety form. Prior to MRI scanning, participants underwent a training and calibration procedure on the mazenavigation task, and on the effort-based decision-making task for a subset of the participants $(\mathrm{n}=19)$. In the MRI scanner, all participants completed the maze-navigation task $(\mathrm{n}=30)$, and two subsets of the participants completed the effort-based decision-making task $(n=19)$ and/or a resting state scan $(n=19)$. After scanning, participants were taken to a separate interview room to complete a debriefing interview. Participants were compensated for their participation upon completion of study procedures.

Imaging data acquired from one participant during the maze-navigation task was excluded prior to analysis due to the individual falling asleep during the task. Thus, the final sample included 29 participants for the maze-navigation task analysis $\left(\mathrm{M}_{\mathrm{age}}=24.41 ; \mathrm{SD}_{\mathrm{age}}=5.43\right.$;

21 female), 19 participants for the effort-based decision-making task $\left(\mathrm{M}_{\mathrm{age}}=24.26 ; \mathrm{SD}_{\mathrm{age}}=4.64\right.$; 14 female), and 19 participants for the resting-state scan $\left(\mathrm{M}_{\mathrm{age}}=24.94 ; \mathrm{SD}_{\mathrm{age}}=5.53 ; 12\right.$ female $)$. 


\section{$\underline{\text { Maze-navigation task }}$}

The task was programmed using Unity 3D (Unity Technologies ApS). A schematic of a single trial is shown in Figure 1A. On each trial, participants completed first-person navigation through a single-path virtual maze in pursuit of monetary rewards. Each trial was associated with one of four maze structures, two of which required a single $90^{\circ}$ turn (left or right), two of which required two $90^{\circ}$ turns (left-then-right or right-then-left), and all of which were equated in approximate navigation time $(\sim 5 \mathrm{~s})$. Regardless of structure, each maze was comprised of $1 \mathrm{x} 1$ unit $^{2}$ floors placed adjacently to form a path, bounded by $1 \mathrm{x} 1$ unit $^{2}$ walls. Participants controlled movement using a 4-button box with the right (i.e., dominant) hand. Specifically, the middle finger button moved the participant forward ( $\mathrm{r}=2.2 \mathrm{units} / \mathrm{s})$, and the index and ring finger buttons each rotated the player clockwise and counterclockwise, respectively $(\omega=0.5 \pi \mathrm{rad} / \mathrm{s})$. Holding down the buttons resulted in continuous movement or rotation (except during the High-Effort condition, as detailed below). Acceleration was applied to player motion to mimic real-life motion. Only one type of motion was allowed at any given moment (i.e., pressing multiple buttons resulted in no motion). The participant view was set at 0.6 units above the floor, with a $10^{\circ}$ tilt along the axis parallel to the ground.

Importantly, each trial was associated with one of four navigation conditions: i) The High-Effort condition required participants to repeatedly press the button associated with forward-motion to advance through the maze. In this condition, each button press moved the participant forward by an incremental distance, and the distance traveled per button press (i.e., effort level) was individually calibrated during the training procedure; ii) The Low-Effort condition required participants to advance through the maze with the default button controls (i.e., simply hold down buttons to move and rotate). This condition served as an active-navigation 
control for the high-effort condition, which allows for examination of effects specifically related to vigor by subtracting out activity associated with mere action; iii) The Passive-Motion condition required participants to view moving through the maze without making any action.

This condition served as a no-action control for the low-effort condition to examine effects related to mere action; and iv) The No-Motion condition required participants to wait for the approximate duration of the maze ( $\sim 4.75 \mathrm{~s})$, after which the participant was teleported to the goal. This condition served as a visual motion control for the Passive-Motion condition. Thus, two of the conditions (High-Effort/Low-Effort) required individuals to actively navigate through the maze, while the other two conditions (Passive-Motion/No-Motion) entailed passive completion of navigation. Participants failed the trial if they did not reach the goal within a liberal time-limit (5.5s) in the active navigation conditions, or if they initiated action in the passive navigation conditions.

Each trial proceeded as follows: (1) Initial Approach phase: At the beginning of each trial, the participant position was initialized to the beginning of a maze, immediately after which participants could initiate movement. (2) Cue phase: Upon reaching a 'trigger' location in the maze, participants were rendered immobile and the floor unit immediately in view changed color for $2 \mathrm{~s}$, before returning to its original texture. The colored cue informed participants to the navigation condition for that trial. (3) Jittered interstimulus interval (ISI): The cue was immediately followed by a jittered fixation period, whereby a '+' was rendered on top of the maze scene for a Poisson-distributed jitter period (2-5s) with a mean duration of 2.5s. (4) Navigation phase: After the fixation cross disappeared, participants regained control of movement and completed navigation according to the navigation condition, as detailed above. On failed trials, participants were presented with a feedback screen for $2 \mathrm{~s}$ indicating that they 
424 failed, followed by an intertrial interval (ITI; skip to (8)). (5) Jittered ISI: Upon successfully

425 reaching the door at the end of the maze (the 'goal'), participants were again rendered immobile

426 for another jitter period (2-5s) with a mean duration of 2.5s. (6) Reward phase: Following the ISI,

427 participants were presented with an animation of the door opening followed by a monetary

428 reward, represented by a dollar amount rendered on the surface of a coin. Each trial was

429 associated with one of 4 bins of reward magnitudes (\$0, $\$ 1.68-2.78, \$ 2.79-\$ 3.89, \$ 3.90-5.00)$,

430 from which an amount was randomly selected. (7) Rating phase: Once every 4 trials, starting on

431 the first trial of each run, the participant was asked to make a mood rating on a Likert-scale

432 between 1 (not happy at all) to 4 (very happy) using button press. (8) Jittered ITI: After reward

433 receipt, failure feedback, or mood rating, participants were presented with a '+' rendered on a

434 grey screen for a duration (2-6s) jittered around $3 \mathrm{~s}$.

Prior to scanning, participants completed a $15 \mathrm{~min}$ training procedure of the maze-

436 navigation task. Participants were introduced to the movement controls, navigation conditions,

437 and trial structure, and informed that a proportion of the reward they obtain on each trial will be

438 added to their compensation as a bonus. Once participants indicated understanding of task

439 instructions, they completed 16 practice trials (4 per navigation condition) on a laptop computer.

440 Participants were instructed to use the same hand and fingers that would be used to perform the

441 task in the MRI scanner.

442

Each participant's average rate of key pressing during the high-effort trials were used to

443 calibrate their effort levels for the in-scanner task. To ensure collection of button pressing rates

444 for sufficient durations of time, the distance traveled per button press in the high-effort condition

445 during the practice was set low (0.11 units/press) and no time-limits were imposed. However,

446 participants were informed that there would be a time-limit for the active navigation conditions 
447 when they complete the task in the scanner and were instructed to complete the mazes during the

448 practice as quickly as possible. From the practice data, the calibrated effort level (i.e., distance, d, 449 per button press) for each participant was calculated as

450

$$
\mathrm{d}=\frac{2.2}{0.7 r}
$$

451

452 where $\mathrm{r}$ is the average key-pressing rate during the first $5.5 \mathrm{~s}$ of the navigation phase (=time-limit 453 on in-scanner trials) in the High-Effort trials. 2.2 corresponds to the default rate of motion

454 (unit/s). $70 \%$ of the average rate was chosen to minimize motion in the scanner, whilst

455 maintaining similar completion times across conditions. All participants successfully completed

456 the practice trials and demonstrated understanding of the four navigation conditions.

During the in-scanner task, the 4 navigation conditions and 4 reward bins were equally

458 distributed and balanced across trials and runs. Each participant completed 3 runs with 32 trials

459 each $(\sim 11 \mathrm{~min} / \mathrm{run})$, and trials were presented in a fixed-randomized order. Participants

460 successfully completed the maze on $93 \pm 2 \%$ of trials, indicating low rates of failure. Importantly,

461 mean completion times across conditions were all within $100 \mathrm{~ms}\left(\mathrm{M}_{\text {High-Effort }}=4.6 \mathrm{~s}\right.$; $\mathrm{M}_{\text {Low- }}$

462 Effort $=4.5 \mathrm{~s}, \mathrm{M}_{\text {Passive-Motion }}=4.7 \mathrm{~s} ; \mathrm{M}_{\mathrm{No}-\mathrm{Motion}}=4.7 \mathrm{~s}$ ), suggesting that condition-based differences in

463 “time-on-task" were unlikely to significantly affect results. Individuals initiated navigation faster

464 on High-Effort trials $(M=550 \mathrm{~ms}$, std=200ms) compared to Low-Effort trials $(\mathrm{M}=630 \mathrm{~ms}$,

$\left.465 \mathrm{std}=210 \mathrm{~ms} ; t_{(28)}=2.55, p=0.02\right)$. Self-reported mood ratings did not differ between the active

466 (High-/Low-Effort) and passive (Passive-/No-Motion) navigation conditions ( $p=0.29 ; \mathrm{M}=2.9$,

$467 \quad \mathrm{std}=0.7)$. 


\section{Effort-based decision-making task}

The task was previously programmed using Psychtoolbox for MATLAB ${ }^{30}$. A schematic of a single trial is shown in Figure 3A. In this task, participants made a series of binary choices provided information about the dollar amount of the reward and the required amount of physical effort to obtain that reward for each choice option. Specifically, participants chose whether to receive $\$ 1.00$ for no work or to complete an effortful task for a larger reward of varying amounts. On each trial, the effortful task option was associated with one of 4 bins of reward magnitude $(\$ 1.25-2.39, \$ 2.40-3.49, \$ 3.50-4.60$, and $\$ 4.61-5.73)$, from which a dollar amount was randomly selected. The effort level was presented as the height of a vertical bar $(20 \%, 50 \%, 80 \%$, or $100 \%$ of the participant's individually-calibrated maximum effort level).

Prior to the scan, participants completed a training and calibration procedure. Calibration was conducted via three independent trials in which participants were asked to repeatedly press a key using their left (i.e., non-dominant) pinky finger as rapidly as possible within 20s. Each participant's maximum effort level was operationalized as the average number of keys pressed across the three trials. Participants then practiced completing the effortful tasks that varied in the required amount of key press $(20,50,80$, and $100 \%$ of their maximum effort level) within a constant time-limit (20s). They completed 4 trials at each level for a total of 16 trials.

Participants were informed that the physical effort component would not be completed inside the MRI scanner, but would be completed immediately following the scan, based on the in-scanner choices. Additionally, participants were instructed that three trials would be randomly selected at the end of the session, from which the reward they earned would be added as a bonus to their compensation. 
Each trial was structured as follows: Participants were first presented with either the

492 reward or effort magnitude of the effortful option (Cue 1) on the left or right half of the screen

493 for a jittered duration $(2-6 \mathrm{~s}$; mean=2.98s). This information remained on the screen while the

494 other piece of information (Cue 2) appeared on the opposite side of the screen for another jittered

495 duration $(2-6 \mathrm{~s}$; mean=3.23s). Participants were then prompted to choose whether to accept the

496 presented effortful task option, or reject the option in favor of receiving $\$ 1.00$ for no work

497 (Choice phase). Participants indicated their choice using button press (index-finger button to

498 accept; middle-finger button to reject), which was then presented on the screen with the words

499 "ACCEPTED" or "REJECTED" according to their choice. Order of information (effort-then-

500 reward vs. reward-then-effort) and the presented side of screen were counterbalanced across

501 trials. Each participant completed 2 runs with 44 trials each $(\sim 9 \mathrm{~min} / \mathrm{run})$, and trials were

502 presented in a fixed-randomized order.

503 After the scan, participants were presented with each of the effortful choice options they

504 accepted during the in-scanner task and performed the associated tasks. For each trial,

505 participants were given the option to change their choice, to examine whether performing the

506 effortful task in real time influenced their willingness to expend effort. We observed that choice

507 behavior was consistent across contexts, such that participants made the same choice on $95 \pm 3 \%$

508 of trials.

509

510 Behavioral and imaging data acquisition

Stimuli were presented via back-projection mirror, and participants completed the maze-

512 navigation and effort-choice task using an MR-compatible 4-button box (Current Designs Inc). 
513 Inflatable pads (Multipad 01; Pearltec AG) placed around participants' heads were used to

514 minimize head motion.

Participants were scanned in a 3-Tesla Siemens TIM Trio scanner (Siemens AG) with a

516 32-channel head-coil using multiband structural and functional imaging ${ }^{54}$. Each session began

517 with a 3-plane localizer scan for slice alignment, and a single-shot, high-resolution structural

518 MPRAGE sequence $(\mathrm{TR} / \mathrm{TE}=1900 / 2.27 \mathrm{~ms}$; flip angle=9; FoV=250x250mm; 192x1.0mm

519 slices). Blood oxygen level dependent functional images were acquired with T2* ${ }^{*}$ weighted EPI

520 sequences with a multiband acceleration factor of $4\left(\mathrm{TR} / \mathrm{TE}=1000 / 30.0 \mathrm{~ms}\right.$; flip angle $=65^{\circ}$;

$521 \quad$ FoV=220x220mm; 52x3.00mm slices).

522

523 Behavioral data analysis

Behavioral data from the maze-navigation task were used to examine latencies to initiate

525 action, rates of navigation completion, durations of navigation completion, and mood ratings.

526 Latency to initiate action was operationalized as the time between the offset of the jittered ISI

527 following the Cue phase and movement onset, and was computed separately for each of the

528 active navigation conditions (High-/Low-Effort). The rate of navigation completion was

529 calculated as the number of successfully-completed trials divided by the total number of trials.

530 Navigation time was calculated separately for each navigation condition, operationalized as the

531 time between the onset of movement and reaching the goal for the active navigation trials (High-

532 (Low-Effort), and between the offset of the jittered ISI following the Cue phase and reaching the

533 goal for the passive navigation trials (Passive-/No-Motion). Self-reported mood ratings were

534 averaged across trials separately across the active navigation conditions (High-/Low-Effort) and

535 across the passive navigation conditions (Passive-/No-Motion). 
Behavioral data from the effort-based decision-making task were used to compute the

537

538 539

540

541 which describes subjective value (SV) as the objective reward magnitude (R) discounted by the

542 subjective cost determined by the effort level (E; between $0-100 \%$ ) and two free parameters, $\mathrm{k}$ 543 and $\mathrm{p}$.

544

$545 \quad$ Imaging data analysis

546

547

548

549

550

551

552

553

554 the influence of strong outliers. Effects in group-level whole-brain analyses were considered 555 significant at a voxelwise threshold of $p<0.0005$ combined with a cluster-extent threshold 555 significant at a voxelwise threshold of $p<0.0005$ combined with a cluster-extent threshold 557 specified.

$$
S V=R-k E^{p}
$$

Functional images from the maze-navigation and effort-based decision-making tasks were preprocessed using SPM12 scripts through NeuroElf v1.1 (neuroelf.net). Specifically, images were co-registered to the structural image, motion-corrected, warped to the MNI template, and smoothed using a Gaussian filter (6mm full width-half maximum). Raw and preprocessed data were subjected to multiple tests for quality assurance and inspected for spiking and motion. Volumes were discarded if the root mean square of motion parameters exceeded a single voxel dimension $(3 \mathrm{~mm})$, or if striping was identified through visual inspection of each functional volume. Subject-level modeling of trial events was conducted using robust regression to reduce 
For the maze-navigation task, the subject-level general linear model (GLM) included the

559 Cue phase, first half of the Navigation phase (Navigation-Start phase), and Reward phase as

560 regressors of interest, with each regressor separated by navigation condition. To examine the

561 effect of reward receipt, the Reward phase was further divided into rewarded (reward $>\$ 0.00$ )

562 and non-rewarded (reward $=\$ 0.00)$ trials. A second GLM included the trial-by-trial reward

563 magnitude as a parametric modulator for the Reward phase, instead of separating the rewarded

564 and non-rewarded trials. These models also included the Initial Approach phase, latter half of the

565 Navigation phase, and Rating phase, as well as the ISIs, to omit their influences on the implicit

566 baseline. In addition, motion parameters and their squares, as well as high-pass filter parameters

567 were included as additional nuisance regressors. Given the potential impact of smoothing on the

568 functional segregation of striatal subregions detected in the above models, two other GLMs were

569 generated using unsmoothed images but otherwise following the same procedures as the first and

570 second GLMs.

571

Group-level contrasts were generated to examine the effects of effort (High-Effort>Low-

572 Effort) and Action (Low-Effort>Passive-Motion) during the Cue and Navigation Start phases,

573 and to examine the effect of reward (Reward $>$ No-Reward, across all conditions) and magnitude

574 of reward (in the parametric models) during the Reward phase. To examine the effect of effort on

575 striatal response to reward, an ROI analysis was conducted by tracking responses within the aVS

576 region found to respond to reward magnitude (left sub-peak: $-6,18$, -6; right sub-peak: 6, 15, -3;

$577 k=77)$ in the parametric model. Then, we extracted beta parameters during reward receipt on

578 rewarded trials in this independently-defined ROI, and a pairwise comparison was conducted

579 between the conditions that required effort (High-/Low-Effort) and those that did not require

580 effort (Passive-/No-Motion). 
To examine the functional selectivity of striatal subregions found during the maze-

582

583

584

585

586

587

588

589

590

591

592

593

594

595

596

597

598

599

600

601

602

603

navigation task, ROI analyses were conducted using a leave-one-subject-out (LOSO) approach to avoid any circularity or non-independence in the definition of ROIs ${ }^{55}$. This was conducted by defining ROIs using group-level contrasts that include data from all but one participant; extracting beta parameters from the removed participant's data; and repeating this procedure for all participants. Beta parameters were extracted from striatal subregions associated with effortinitiation (Navigation-Start phase, High-Effort>Low-Effort), action initiation (Navigation-Start phase, Low-Effort>Passive-Motion), and reward receipt (Reward phase, reward $>$ no-reward, across all conditions). Effects of effort, action, and reward for each subregion was calculated by subtracting beta parameters according to the relevant contrasts (Effort: High-Effort - LowEffort; Action: Low-Effort - Passive-Motion; Reward: Reward - No-Reward, averaged across navigation conditions). A 2 Region (dmVS, putamen) *2 Effect (Effort, Action) repeatedmeasures ANOVA was conducted to test for functional dissociation between dmVS and VS, and to examine whether dmVS activity was selective to effort and not mere action. Additionally, a 2 Region (dmVS, VS) * 2 Effect (Effort, Reward) repeated-measures ANOVA was conducted to test for functional dissociation between these neighboring regions associated with effort and reward.

Additionally, to examine the presence of both effort-activation and effort-discounting effects within a VS region, we functionally-defined a VS ROI (peak: $6,0,-9 ; k=72$ ) that responded to reward after completing effortful navigation (Reward $>$ No-Reward, High-Effort condition). This reward-sensitive ROI specific to the High-Effort condition was defined at a more lenient voxelwise $p<0.005$ given that this region was necessarily defined on only $25 \%$ of the data. We note that this threshold was used for ROI definition only and not for subsequent 
604

605

606

607

608

609

610

611

612

613

614

615

616

617

618

619

620

621

622

623

624

625

626

inferential analyses. Beta parameters from this independently-defined ROI were extracted from the High-Effort and Low-Effort conditions during the Navigation Start and Reward phases, and a 2 Condition (High-Effort, Low-Effort) * 2 Phase (Navigation Start, Reward) repeated-measures ANOVA was conducted. Pairwise comparisons were conducted to parse out the nature of significant effects.

The CONN toolbox was used for resting-state fMRI data analysis. Preprocessing of images included motion correction, co-registration to structural scan, MNI normalization, and smoothing using an 8mm Gaussian smoothing kernel. Striatal subregions were functionallydefined from the maze-navigation data and used as seed regions to create individual seed-tovoxel fcMRI maps. Specifically, the seed regions included a bilateral dmVS region associated with effort initiation (Navigation Start phase, High-Effort>Low-Effort), a region in bilateral putamen associated with action (Navigation Start phase, Low-Effort $>$ Passive-Motion), and a bilateral VS region associated with reward (Reward phase, parametric effect of reward magnitude, across all conditions). For each of the three fcMRI maps, comparison maps were generated using the spatially-corresponding subregions among the previously-reported connectivity-based parcellation of the striatum (regions 4, 5, and 7 in the seven subregion parcellation $)^{47}$. Additionally, fcMRI maps using striatal regions defined from the mazenavigation task were computed using an independent set of 7 T resting-state fMRI data $(\mathrm{N}=22)^{49}$ downloaded from OpenNeuro (openneuro.org). Specifically, two runs per subject of whole-brain resting-state data from session one in the dataset were included for the purpose of our study.

Processing of these data followed the same procedures through CONN as described above, except for using a $6 \mathrm{~mm}$ smoothing kernel to account for smaller voxel size. Further, SørensenDice indices were calculated for each ROI defined in the maze-navigation task and the 
627 corresponding connectivity-based $\mathrm{ROI}^{47}$ to test for spatial similarity between the ROI definitions.

628 To do this, we used the nilearn - http://nilearn.github.io - library to resample the image space

629 and affine of task-based ROI masks on the connectivity-based ROI masks.

630 For the effort-based decision-making task, the first subjective-level GLM included the

631 Cue 1 and Cue 2 phases as regressors of interests, with Cue 1 regressors separated by type of

632 information presented (effort vs. reward). We also included the Choice and Rating phases to omit

633 their influence on the implicit baseline. Again, motion parameters and their squares, as well as

634 high-pass filter parameters were included as additional nuisance regressors. At the group-level,

635 we examined regions in which activity was higher during Cue 2 compared to Cue 1 (Cue 2>Cue

636 1). To examine the effect of subjective value during the valuation period, we additionally

637 included the trial-by-trial SV of the chosen option as a parametric regressor for Cue 2 in a second

638 GLM. At the group-level, we focused on the negative parametric effects of SV to identify

639 regions that increased in response to decreasing value (e.g., higher effort costs). ROI analyses

640 were conducted to examine the effect of negative SV in the dACC and VS. To do this, the dACC

641 ROI (left sub-peak: -10, 26, 32; right sub-peak: 10, 24, 36; $k=334$ ) was functionally-defined

642 using the same parametric analysis (effect of negative SV) from our prior study using this task

64327,30 at $p<0.05$ familywise-error corrected with voxelwise $p<0.0005$, and the VS ROI was defined

644 using an anatomical nucleus accumbens mask (left peak: -10, 10, -8, k=309; right peak: 10, 10, -

$6458, \mathrm{k}=310)$. A pairwise comparison was conducted on the extracted parametric effects of negative

646 SV within the dACC and VS.

647

648

649
Multivariate pattern analysis (MVPA) of the effort-based decision making task was

performed using the scikit-learn ${ }^{56}$ and nilearn libraries. We trained linear support vector

machines with the $\mathrm{C}$ parameter fixed at a value of 1 . Each classifier used unsmoothed functional 
650 images as features to predict the type of information (effort or reward) shown at Cue 1. For each

651 trial, we selected four fMRI volumes offset by four seconds from the onset of Cue 1 presentation.

652 Classifiers were trained and evaluated at the single-subject level using a four-fold cross

653 validation procedure in which data were randomly partitioned into four subsets. To avoid

654 temporal confounds, all volumes within a trial were kept in the same fold. The accuracies

655 reported are the average of the four folds. For each subject, we tested three classifiers that

656 differed in the spatial filtering of the images used as inputs. The first model used whole-brain

657 images as inputs; feature selection was then performed by selecting 1,000 voxels that were most

658 strongly associated with the training labels based on their ranking of ANOVA F-values. In the

659 second and third models, inputs were constrained to voxels within the aVS and dmVS masks,

660 respectively. To test the significance of classifier performance, we ran permutation tests in which

661 the same classification procedure was repeated using 1,000 random permutations of training

662 labels ${ }^{57}$. This provided a distribution of chance-level accuracy; we consider the performance of a

663 classifier as significantly above chance if is greater than $95 \%$ of accuracies obtained using

664 permutated tests ${ }^{57}$.

665

Additionally, to examine whether individual differences in striatal responses during maze-navigation predicted ventral striatal signals in the effort-based decision-making task, we

667 used the extracted beta parameters from the dmVS (obtained from Navigation Start phase, High-

668 Effort $>$ Low-Effort) and VS (obtained from Reward phase, Reward $>$ No-Reward, High-Effort

669 condition) to compute individual differences in the effect of effort (High-Effort - Low-Effort)

670 during effort initiation for $\mathrm{dmVS}$ and during reward receipt for VS. We then correlated these

671 effects with extracted beta parameters from the VS during an open contrast of the presentation of

672 effort information at Cue 1 (averaged across all presented effort levels) in the effort-based 
673 decision-making task. An open contrast (i.e., contrasted against the implicit baseline) was used

674 as we felt this best represented neural responses to potential effort as compared to the default "no

675 effort" option that was always available to participants on this task.

676

677 


\section{References}

6791 Bartra, O., McGuire, J. T. \& Kable, J. W. The valuation system: a coordinate-based meta680 analysis of BOLD fMRI experiments examining neural correlates of subjective value.

$681 \quad$ Neuroimage 76, 412-427 (2013).

6822 Mogenson, G. J., Jones, D. L. \& Yim, C. Y. From motivation to action: functional 683 interface between the limbic system and the motor system. Progress in neurobiology 14, $68469-97(1980)$.

6853 Knutson, B., Delgado, M. R. \& Phillips, P. E. in Neuroeconomics 389-406 (Elsevier, 686 2009).

6874 Berke, J. D. What does dopamine mean? Nature neuroscience 21, 787-793 (2018).

6885 Berridge, K. C. \& Robinson, T. E. What is the role of dopamine in reward: hedonic 689 impact, reward learning, or incentive salience? Brain research reviews 28, 309-369 $690 \quad$ (1998).

6916 Schultz, W., Carelli, R. M. \& Wightman, R. M. Phasic dopamine signals: from subjective 692

693

694 154 (2015). Salamone, J. D., Correa, M., Farrar, A. \& Mingote, S. M. Effort-related functions of

6978 Wise, R. A. Dopamine, learning and motivation. Nature reviews neuroscience 5, 483

698 (2004).

9 McClure, S. M., Laibson, D. I., Loewenstein, G. \& Cohen, J. D. Separate neural systems value immediate and delayed monetary rewards. Science 306, 503-507 (2004).

701 Wittmann, M., Leland, D. S. \& Paulus, M. P. Time and decision making: differential contribution of the posterior insular cortex and the striatum during a delay discounting task. Experimental Brain Research 179, 643-653 (2007).

11 Gregorios-Pippas, L., Tobler, P. N. \& Schultz, W. Short term temporal discounting of reward value in human ventral striatum. Journal of neurophysiology 101, 1507-1523

706

707 (2009).

708 Kable, J. W. \& Glimcher, P. W. The neural correlates of subjective value during intertemporal choice. Nature neuroscience 10, 1625 (2007). 15734 (2009). valuation subsystems for delay and effort decision costs. Journal of Neuroscience 30, 14080-14090 (2010). function of reward probability is coded in human nucleus accumbens. Neuroimage 31, 790-795 (2006). errors of prediction in the human brain. Journal of Neuroscience 26, 9530-9537 (2006).

17 Levy, D. J. \& Glimcher, P. W. The root of all value: a neural common currency for choice. Current opinion in neurobiology 22, 1027-1038 (2012). 
72218 Croxson, P. L., Walton, M. E., O'Reilly, J. X., Behrens, T. E. \& Rushworth, M. F. Effortbased cost-benefit valuation and the human brain. Journal of Neuroscience 29, 45314541 (2009).

19 Kurniawan, I. T. et al. Choosing to make an effort: the role of striatum in signaling physical effort of a chosen action. Journal of neurophysiology 104, 313-321 (2010).

20 Schmidt, L., Lebreton, M., Cléry-Melin, M.-L., Daunizeau, J. \& Pessiglione, M. Neural mechanisms underlying motivation of mental versus physical effort. PLoS biology 10, e1001266 (2012).

21 Burke, C. J., Brünger, C., Kahnt, T., Park, S. Q. \& Tobler, P. N. Neural integration of risk and effort costs by the frontal pole: only upon request. Journal of Neuroscience 33, 17061713 (2013).

22 Kurniawan, I. T., Guitart-Masip, M., Dayan, P. \& Dolan, R. J. Effort and valuation in the brain: the effects of anticipation and execution. Journal of Neuroscience 33, 6160-6169 (2013).

23 Skvortsova, V., Palminteri, S. \& Pessiglione, M. Learning to minimize efforts versus maximizing rewards: computational principles and neural correlates. Journal of Neuroscience 34, 15621-15630 (2014).

24 Massar, S. A., Libedinsky, C., Weiyan, C., Huettel, S. A. \& Chee, M. W. Separate and overlapping brain areas encode subjective value during delay and effort discounting. Neuroimage 120, 104-113 (2015).

25 Scholl, J. et al. The good, the bad, and the irrelevant: neural mechanisms of learning real and hypothetical rewards and effort. Journal of Neuroscience 35, 11233-11251 (2015).

26 Bonnelle, V., Manohar, S., Behrens, T. \& Husain, M. Individual differences in premotor brain systems underlie behavioral apathy. Cerebral cortex 26, 807-819 (2015).

27 Klein-Flügge, M. C., Kennerley, S. W., Saraiva, A. C., Penny, W. D. \& Bestmann, S. Behavioral modeling of human choices reveals dissociable effects of physical effort and temporal delay on reward devaluation. PLoS computational biology 11, e1004116 (2015).

28 Chong, T. T.-J. et al. Neurocomputational mechanisms underlying subjective valuation of effort costs. PLoS biology 15, e1002598 (2017).

29 Hauser, T. U., Eldar, E. \& Dolan, R. J. Separate mesocortical and mesolimbic pathways encode effort and reward learning signals. Proceedings of the National Academy of Sciences 114, E7395-E7404 (2017).

30 Arulpragasam, A. R., Cooper, J. A., Nuutinen, M. R. \& Treadway, M. T. Corticoinsular circuits encode subjective value expectation and violation for effortful goal-directed behavior. Proceedings of the National Academy of Sciences 115, E5233-E5242 (2018).

31 Aridan, N., Malecek, N. J., Poldrack, R. A. \& Schonberg, T. Neural correlates of effortbased valuation with prospective choices. Neuroimage 185, 446-454 (2019).

32 Endepols, H. et al. Effort-based decision making in the rat: an [18F] fluorodeoxyglucose micro positron emission tomography study. Journal of Neuroscience 30, 9708-9714 (2010).

33 Cousins, M. S. \& Salamone, J. D. Nucleus accumbens dopamine depletions in rats affect relative response allocation in a novel cost/benefit procedure. Pharmacology Biochemistry and Behavior 49, 85-91 (1994).

34 Floresco, S. B. The nucleus accumbens: an interface between cognition, emotion, and action. Annual review of psychology 66, 25-52 (2015). 
76735 da Silva, J. A., Tecuapetla, F., Paixão, V. \& Costa, R. M. Dopamine neuron activity before action initiation gates and invigorates future movements. Nature 554, 244 (2018). Day, J. J., Jones, J. L., Wightman, R. M. \& Carelli, R. M. Phasic nucleus accumbens dopamine release encodes effort-and delay-related costs. Biological psychiatry 68, 306309 (2010).

38 Syed, E. C. et al. Action initiation shapes mesolimbic dopamine encoding of future rewards. Nature neuroscience 19, 34-39 (2016).

39 Lau, B. \& Glimcher, P. W. Action and outcome encoding in the primate caudate nucleus. Journal of Neuroscience 27, 14502-14514 (2007).

40 Samejima, K., Ueda, Y., Doya, K. \& Kimura, M. Representation of action-specific reward values in the striatum. Science 310, 1337-1340 (2005).

41 Zaborszky, L. et al. Cholecystokinin innervation of the ventral striatum: a morphological and radioimmunological study. Neuroscience 14, 427-453 (1985).

42 Penner, M. R. \& Mizumori, S. J. Neural systems analysis of decision making during goaldirected navigation. Progress in neurobiology 96, 96-135 (2012).

43 Di Chiara, G. et al. Dopamine and drug addiction: the nucleus accumbens shell connection. Neuropharmacology 47, 227-241 (2004).

44 Van Der Plasse, G., Schrama, R., Van Seters, S. P., Vanderschuren, L. J. \& Westenberg, H. G. Deep brain stimulation reveals a dissociation of consummatory and motivated behaviour in the medial and lateral nucleus accumbens shell of the rat. PloS one 7, e33455 (2012).

45 Parkinson, J. A., Willoughby, P. J., Robbins, T. W. \& Everitt, B. J. Disconnection of the anterior cingulate cortex and nucleus accumbens core impairs Pavlovian approach behavior: Further evidence for limbic cortical-ventral striatopallidal systems. Behavioral neuroscience 114, 42 (2000).

46 Ko, D. \& Wanat, M. J. Phasic dopamine transmission reflects initiation vigor and exerted effort in an action-and region-specific manner. Journal of Neuroscience 36, 2202-2211 (2016).

47 Choi, E. Y., Yeo, B. T. \& Buckner, R. L. The organization of the human striatum estimated by intrinsic functional connectivity. Journal of neurophysiology 108, 22422263 (2012).

48 Engelhard, B. et al. Specialized coding of sensory, motor and cognitive variables in VTA dopamine neurons. Nature, 1 (2019).

49 Gorgolewski, K. J. et al. A high resolution 7-Tesla resting-state fMRI test-retest dataset with cognitive and physiological measures. Scientific data 2, 140054 (2015).

50 Howe, M. W., Tierney, P. L., Sandberg, S. G., Phillips, P. E. \& Graybiel, A. M. Prolonged dopamine signalling in striatum signals proximity and value of distant rewards. Nature 500, 575-579 (2013).

51 Roesch, M. R., Singh, T., Brown, P. L., Mullins, S. E. \& Schoenbaum, G. Ventral striatal neurons encode the value of the chosen action in rats deciding between differently delayed or sized rewards. Journal of Neuroscience 29, 13365-13376 (2009). 
influences on instrumental behaviour. European Journal of Neuroscience 13, 1984-1992 (2001).

81453 Sonkusare, S., Breakspear, M. \& Guo, C. Naturalistic Stimuli in Neuroscience: Critically

815

81654 Van Essen, D. C. et al. The WU-Minn human connectome project: an overview. Acclaimed. Trends in Cognitive sciences 23, 699-714 (2019).

$817 \quad$ Neuroimage 80, 62-79 (2013).

$81855 \quad$ Esterman, M., Tamber-Rosenau, B. J., Chiu, Y.-C. \& Yantis, S. Avoiding non-

82156 Pedregosa, F. et al. Scikit-learn: Machine learning in Python. Journal of machine

$822 \quad$ learning research 12, 2825-2830 (2011).

82357 Ojala, M. \& Garriga, G. C. Permutation tests for studying classifier performance. Journal of Machine Learning Research 11, 1833-1863 (2010). 